\title{
An Advertising Spreading Model for Social Networks
}

\author{
Jing $\mathrm{Yi}^{\mathrm{a}, \mathrm{b}}$, Peiyu Liu ${ }^{\mathrm{a}, *}$, Wenfeng $\mathrm{Liu}^{\mathrm{a}, \mathrm{c}}$, Jingang Ma ${ }^{\mathrm{d}}$, and Tianxia Song ${ }^{\mathrm{a}}$ \\ ${ }^{a}$ School of Information Science and Engineering, Shandong Normal University, Jinan, 250014, China \\ ${ }^{b}$ School of Computer Science and Technology, Shandong Jianzhu University, Jinan, 250101, China \\ 'Department of Computer and Information Engineering, Heze University, Heze, 274015, China \\ ${ }^{d}$ Polytechnic Institute, Shandong University of Traditional Chinese Medicine, Jinan, 250355, China
}

\begin{abstract}
The SIR spreading model cannot fully reflect the regularity of information propagation for social networks. In this paper, based on the influence analysis on the propagation mechanism and network parameters on the process of advertising spreading in social networks, the advertising spreading model that is applied to social networks is established and corresponding dynamic evolution equations are given. Meanwhile, because there is currently no unified evaluation criteria for the validity of spreading models, the application of AEI, which is the advertising effectiveness index used to evaluate and analyze the effectiveness of spreading models, is put forward in this paper. The simulation results demonstrate that the model proposed in this paper can correctly reflect the trend of advertising spreading in social networks and accurately describe the spreading process. The validity of the model is also verified in this paper.
\end{abstract}

Keywords: social network; SIR model; advertising spreading model; AEI index

(Submitted on February 27, 2018; Revised on April 3, 2018; Accepted on May 12, 2018)

(C) 2018 Totem Publisher, Inc. All rights reserved.

\section{Introduction}

In recent years, with the rapid development of information technology, social networks as new social communicating tools have been recognized by more and more people. There are millions of users in some well-known social networking companies, such as Facebook, MySpace and campus networks, etc.. People are increasingly using them to play games, make new friends and spread information. As opposed to the traditional method of information propagation, social networks revolve around people and rely on the interactions between people and information. In social networks, a common phenomenon is to transmit information between friends, in which advertising has been widely used as information embedment. Due to the low cost, wide coverage and rapid spread, this advertising propagation has been widely used. Therefore, it is valuable to study the spreading of advertising information in social networks to maximize the effectiveness of advertising.

Currently, there are several advertising spreading forms, such as node propagation, multi-level core-edge propagation, and circle propagation [2]. Research on the effect of propagation mostly looks at the aspects of sense of identity, brand-loyalty, enhancement of advertising entrance, etc $[2,5,16]$. However, there are a few studies on the process of advertising information propagation in networks, but there is no unified standard for the evaluation of advertising effectiveness. Research on the propagation model mainly focus on the improvement of the SIR and SEIR models $[1,6,7,10,11,12,14,15,17,18,19]$. In Reference [17], an information propagation model based on online social networks is constructed, dynamic evolution equations are obtained, and the propagation rules are analyzed. In Reference [18], the information spreading model of online social networks is analyzed as well as the behavior of nodes with different degrees in social network propagation. In Reference $[1,3,6,7,10,11,12,13,14,15,19,20,21]$, the different improvement strategies of the model under different application backgrounds are discussed, especially with the rumor spreading model $[3,11,13,20,21]$ and online social networks $[4,8,9]$. All of these studies focus on improving the propagation model. There is no uniform index to verify the validity of the improved model.

\footnotetext{
* Corresponding author.

E-mail address: yjzyj2006@163.com
} 
In this paper, using advertising spreading in social networks as the application background and the SIR model of infectious diseases as the basic model [11], advertising promotion models in social networks are analyzed, and a social network advertising promotion model is established. Through the analysis of advertising spreading in social networks, a simulation experiment of the user's behavior is carried out to verify that the proposed model is in line with the characteristics of information propagation in social networks. In order to quantitatively evaluate the current models, the international-popular advertising effectiveness index (AEI) is applied to determine the effectiveness of the propagation model and provide a reference for further study of the effectiveness of the propagation model. Finally, a numerical simulation experiment is carried out.

\section{Model establishments}

\subsection{Analysis on advertising promotion models in social network}

In social networks, users can be regarded as one of the nodes, and users-concerned relationships can be regarded as the edges in the network. Information spreads along the edges between the nodes, and values of the edges represent the intimacy among users. Figure 1 shows a social network that contains 11 points (users), 13 edges, and the closeness of each point.

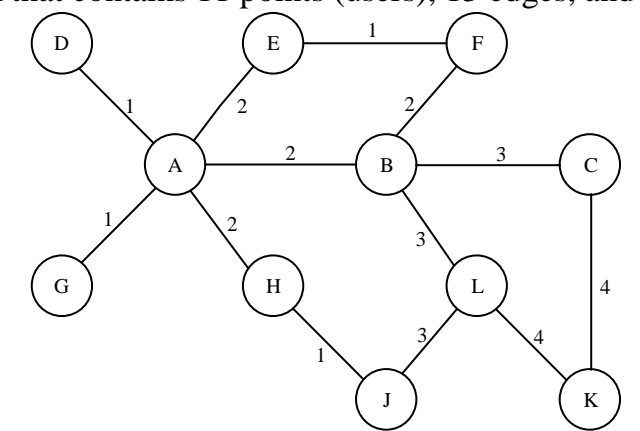

Figure 1. Pattern of information propagation in social networks

In Figure 1, when User A has access to advertising information, Users B, D, E, G, H can also obtain this information, among which Users B, E, G are interested in the content of the advertisement and transmit the information with a certain probability. Thus, they become "disseminators". Users D and H are not interested in the content of the advertising information, and so they won't transmit it. Thus, they become "direct-recovery" to the information. After User G transmits the information to J, he loses interest and never retransmits it. Then, he becomes "the recovery".

In addition, it has been proven in practice that advertising does not follow the mantra "the more, the better". John Philip Jones has proven that high-density advertising coverage is wasteful. Instead, the behaviors that follow can produce more benefits, such as covering for a long time to spread and disperse advertising, advertising in every media outlet in the way that constant dripping wears a stone, advertising in more media to spread and disperse, or advertising in a wider geographical area [2].

\subsection{AEI advertising effectiveness index}

AEI (Advertising Effectiveness Index) refers to the number that is used to quantify the effectiveness of ads. It can be calculated as follows: the number of people who see ads and purchase minus the number of people without the influence of ads. Then, the number of people who are advertising-aroused is determined, and the number is divided by the total number of people surveyed. Then, the result is the advertising effectiveness index. AEI is currently the most popular advertising evaluation index in the world.

The major purpose of advertising is to stimulate consuming behavior, and thus, a focus on the effectiveness of the advertising propagation model will measure the impact of advertising on consuming behavior. In this paper, AEI is used as an index to measure the effectiveness of the model in order to determine whether consuming behaviors are affected by website advertisements. The formula to calculate AEI is:

$$
A E I=\frac{A-(A+C) \times \frac{B}{(B+D)}}{N}
$$


Among them,

A: the number of people who have seen the advertisement and purchased;

B: the number of people who have not seen the advertisement and purchased;

$\mathrm{C}$ : the number of people who have seen the advertisement and did not purchase;

D: the number of people who have neither seen the advertisement nor purchased.

For example, after launching new products for half a month, a company did a market survey, and the total sample number is 50 people, among which the number of people who saw the ad and purchase is 12; the number of people who did not see the ad but purchased is 7; the number of people who saw the ad but did not purchase is 17; the number of people who neither saw the ad nor purchased is 14 ; so AEI $=[12-(12+17) * 7 /(7+14)] / 50=4.7 \%$.

\subsection{Social network advertising spreading model based on AEI}

The SIR model is a fully connected regular network, in which the nodes are connected in accordance with certain rules, and there is orderly topology as well as relatively simple evolution laws. However, research indicates that social networks have complex characteristics, such as microcosm, scale-free and isomerism, etc [17]. Therefore, the SIR model in regular networks cannot faithfully reflect the rules of information propagation in social networks. Research on the improved SIR model mainly focused on increasing users' weight [10], increasing the state nodes [1,12,15,19] and increasing transforming probability $[6,7,10]$ according to specific application occasions. In this paper, with reference to AEI, an improved SIR model is utilized to build a advertising spreading model in social networks.

In the advertising spreading model, the susceptible node $\mathrm{S}$ represents individuals who neither know the ad nor purchase; the spreading node I represent individuals who are aware of the ad and have interest in disseminating and purchasing; the recovery node $\mathrm{R}$ represents individuals who are aware of the ad but lose interest in purchasing or transmitting. From the analysis of section 2.1, we can give some definitions of the probability of advertising spreading in social networks.

Definition 1 A susceptible node $S$ that neither receives the ad nor purchases has contact with a spreading node I. Then, it is transformed into a spreading node; this probability is called $\mathrm{P}_{\mathrm{SI}}$, namely, the internal infection probability.

Definition 2 A spreading node I is transformed into a recovery node $\mathrm{R}$ (which means it loses interest in purchasing or information spreading) after information propagation; accordingly, this probability is called $\mathrm{P}_{\mathrm{IR}}$, namely, the recovery probability.

Definition 3 A node $S$ that is not aware of the ad then acquires information from external media, but is still not interested in it and will never buy it. In this circumstance, it turns into a recovery node $\mathrm{R}$, and the probability is called $\mathrm{P}_{\mathrm{SR}}$, namely, the direct recovery probability.

Definition 4 A node who is not aware of the ad and does not purchase the goods before acquiring information from external media, but then purchases; such a probability is called $\alpha$, namely, external recovery probability.

Through the above analysis, it can be seen that the state of each node in social networks can be converted to $\mathrm{S}, \mathrm{I}$, R in a certain probability to a certain topic of information, and the evolution rules of information propagation model based on SIR in social networks can be described as:

(1) Suppose that in the process of information propagation, the total number $\mathrm{N}$ in the network is the same without considering users moving in and out of the network;

(2) Suppose that $\mathrm{N}(\mathrm{t})$ is the total number of nodes at the time of $\mathrm{t}$ in the social network, and $\mathrm{S}(\mathrm{t})$, $\mathrm{I}(\mathrm{t})$ and $\mathrm{R}(\mathrm{t})$ respectively represent the proportion (i.e. density) of susceptible nodes (that neither receive this ad nor purchase), spreading nodes (that know the ad and purchase), and recovery nodes (that know the ad but do not purchase) at the time of t in the social network. Accordingly, $\mathrm{S}(\mathrm{t})+\mathrm{I}(\mathrm{t})+\mathrm{R}(\mathrm{t})=1$;

(3) A susceptible node $\mathrm{S}$ is transformed into a spreading node I (continues to purchase and spread) with the probability of internal infection $\mathrm{P}_{\mathrm{SI}}$;

(4) A susceptible node S is transformed into a spreading node I (continues to purchase and spread) with the probability of external infection $\alpha$; 
(5) A spreading node I is transformed into the recovery node R (no longer purchases or spreads) after purchasing the product with the recovery probability $\mathrm{P}_{\mathrm{IR}}$;

(6) Although it gets the advertising information, a susceptible node S is directly transformed into the recovery node (no longer buys) with the direct recovery probability $\mathrm{P}_{\mathrm{SR}}$;

(7) All nodes or a certain number of nodes in the network are in the recovery state, or the state of absorption in the network, in which the nodes will never change their states anymore.

According to the above state-transition rules, the state evolution of the improved SIR advertising spreading model is shown in Figure 2.

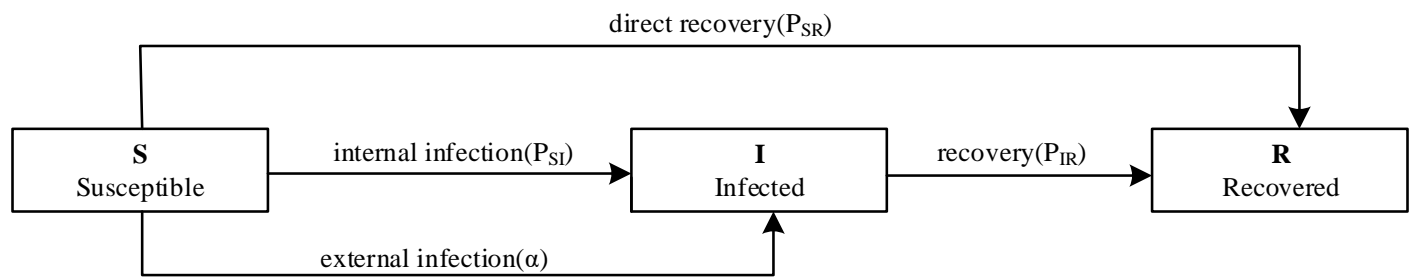

Figure 2. Evolution diagram of the improved SIR advertising spreading model

Using the mean field theory, the dynamic equation of the model is shown in Figure 2:

$$
\left\{\begin{array}{l}
\frac{d S(t)}{d t}=-P_{S I} S(t) I(t)-\alpha S(t)-P_{S R} S(t) \\
\frac{d I(t)}{d t}=P_{S I} S(t) I(t)+\alpha S(t)-P_{I R} I(t) \\
\frac{d R(t)}{d t}=P_{S R} S(t)+P_{I R} I(t) \\
S(t)+I(t)+R(t)=1
\end{array}\right.
$$

\section{Model analyses}

The social network is different from the homogeneous network, and there is a correlation between the degree of the nodes in the social network. Therefore, based on the social network and considering the connectivity between nodes, we need to further analyze Formula 2.

Suppose that $N(k, t)$ is the total number of nodes with $k$ degree, and $S(k, t), I(k, t)$ and $R(k, t)$ respectively represent the proportion (i.e. density) of susceptible nodes (that neither receive the ad nor purchase goods), transmitting nodes (that know the ad and purchase), and recovery nodes (that know the ad but do not purchase) with k degree at time in the social network. Accordingly, $\mathrm{S}(\mathrm{k}, \mathrm{t})+\mathrm{I}(\mathrm{k}, \mathrm{t})+\mathrm{R}(\mathrm{k}, \mathrm{t})=1$. Then, Formula 2 can be further modified into:

$$
\left\{\begin{array}{l}
\frac{d S(k, t)}{d t}=-P_{S I} k S(k, t) \theta(t)-\alpha S(k, t)-P_{S R} S(k, t) \\
\frac{d I(k, t)}{d t}=P_{S I} k S(k, t) \theta(t)+\alpha S(k, t)-P_{I R} I(k, t) \\
\frac{d R(k, t)}{d t}=P_{S R} S(k, t)+P_{I R} I(k, t) \\
S(k, t)+I(k, t)+R(k, t)=1
\end{array}\right.
$$

In Formula 3, $\theta(t)$ is the probability that any edge in the network is connected with spreading node I at t time, namely:

$$
\theta(t)=\frac{\sum_{k} k P(k) I(k, t)}{<k>}
$$

where $\mathrm{P}(\mathrm{k})$ is the degree distribution function of the social network, and $\langle\mathrm{k}\rangle$ is the average node degree of the network. 
In this paper, $\mathrm{N}_{\mathrm{k}, \mathrm{S}}(\mathrm{t})$ is used to express the number of susceptible nodes with k degree at $\mathrm{t}$ time, then $D=\sum_{k} N_{k, S}(t)$; $\mathrm{N}_{\mathrm{k}, \mathrm{I}}(\mathrm{t})$ is used to express the number of infection nodes with $\mathrm{k}$ degree at $\mathrm{t}$ time, which contains two parts: one is the number of nodes infected externally and the other is the number of internal infections. Thus, $A+B=\sum_{k} N_{k, I}(t) ; \mathrm{N}_{\mathrm{k}, \mathrm{R}}(\mathrm{t})$ is used to express the number of recovery nodes with $\mathrm{k}$ degree at $\mathrm{t}$ time, then $C=\sum_{k} N_{k, R}(t)$; so, $\mathrm{N}_{\mathrm{k}, \mathrm{S}}(\mathrm{t})+\mathrm{N}_{\mathrm{k}, \mathrm{I}}(\mathrm{t})+\mathrm{N}_{\mathrm{k}, \mathrm{R}}(\mathrm{t})=\mathrm{N}_{\mathrm{k}}$, in which $\mathrm{N}_{\mathrm{k}}$ is the total number of nodes with $\mathrm{K}$ degree, and $A+B+C+D=\sum_{k} N_{k}$. Based on the analysis of section 2.3 , the dynamic evolution equation of the proposed model is obtained:

$$
\left\{\begin{array}{l}
N_{k, S}(t+1)=N_{k, S}(t)-P_{S I} N_{k, S}(t) N_{k, I}(t) p-\alpha N_{k, S}(t)-P_{S R} N_{k, S}(t) \\
N_{k, I}(t+1)=N_{k}-N_{k, S}(t+1)-N_{k, R}(t+1) \\
N_{k, R}(t+1)=N_{k, R}(t)+P_{S R} N_{k, S}(t)+P_{I R} N_{k, I}(t) p \\
N_{k, S}(t)+N_{k, I}(t)+N_{k, R}(t)=N_{k}(t)
\end{array}\right.
$$

in which $\mathrm{P}$ is the probability that a node is connected to a node with $\mathrm{k}$ degree in the network at $\mathrm{t}$ time, namely:

$$
p=\frac{\sum_{k} k P(k)}{<k>}
$$

and $\mathrm{P}(\mathrm{k})$ is the degree distribution function of the social network, and $\langle\mathrm{k}\rangle$ is the average node degree of the network.

\section{Experimental analyses}

\subsection{Experimental data set}

The Facebook user data set used in this paper consists of 4039 nodes and 88234 edges. With the use of the social network analysis software, Ucinet, the topological characteristics of the network can be obtained as follows: average degree $\langle\mathrm{k}\rangle=78.92$, which means each node in the network has nearly 80 neighbor nodes; maximum node degree is 638 , which means some nodes in the social network have up to 638 neighbor nodes; average clustering coefficient is 0.2056 , which means the relationship among nodes is quite close; number of community is 3 , and the module is 0.2127 , indicating that the network has a clear community structure; average path is 4.71 , indicating that the network has obvious microcosm characteristics.

\subsection{Simulation results and analyses}

In this paper, Matlab simulation software is used to analyze the advertising communication model in social networks given in Formula 3, and the simulation results are analyzed accordingly.

\subsubsection{The varying density of different nodes at different time}

Suppose that there is only one spreading node at the initial time, the other nodes are all susceptible in the social network, and the parameters in the formula are set as follows: $\mathrm{p}=0, \mathrm{P}_{\mathrm{SI}}=0.3$, external infection probability $\alpha=0.2$, recovery probability $\mathrm{P}_{\mathrm{IR}}=0.1$, and direct recovery probability $\mathrm{P}_{\mathrm{SR}}=0.1$. The interrelationship that the density of susceptible nodes, spreading nodes and recovery nodes differ at different times are respectively presented by $\mathrm{S}(\mathrm{t}) 、 \mathrm{I}(\mathrm{t})$ and $\mathrm{R}(\mathrm{t})$, namely:

$$
\begin{aligned}
& S(t)=\sum_{k} k S(k, t) \\
& I(t)=\sum_{k} k I(k, t) \\
& R(t)=\sum_{k} k R(k, t)
\end{aligned}
$$


In Figure 3, the densities of the three types of nodes in the advertising communication process change over time, and the number of experimental iterations is up to 100 times. Figure 3 clearly shows the varying dense curve of susceptible nodes, spreading nodes as well as recovery nodes. It is easy to find that the density of susceptible nodes $\mathrm{S}(\mathrm{t})$ keeps decaying until it comes to nearly 0 ; however, the density of immune nodes $\mathrm{R}(\mathrm{t})$ shows a rapid growth in the initial stage of the topic spreading, and gradually stabilizes after reaching its peak value, and then it finally tends to 1 .

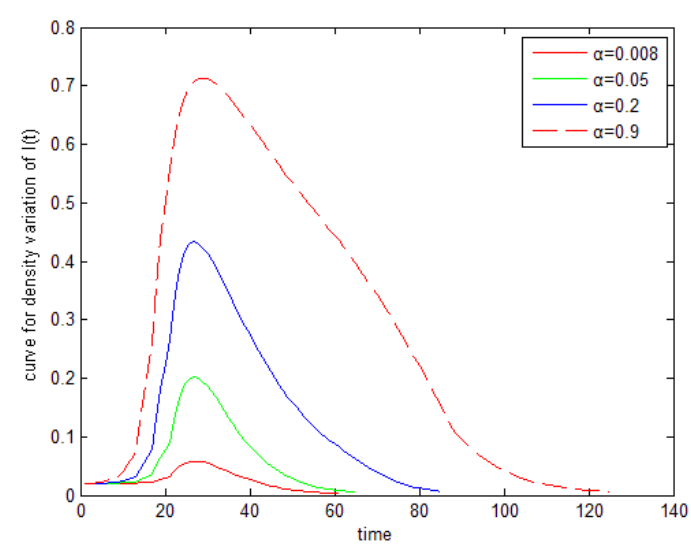

Figure 3. Tendency for node density in different states over time

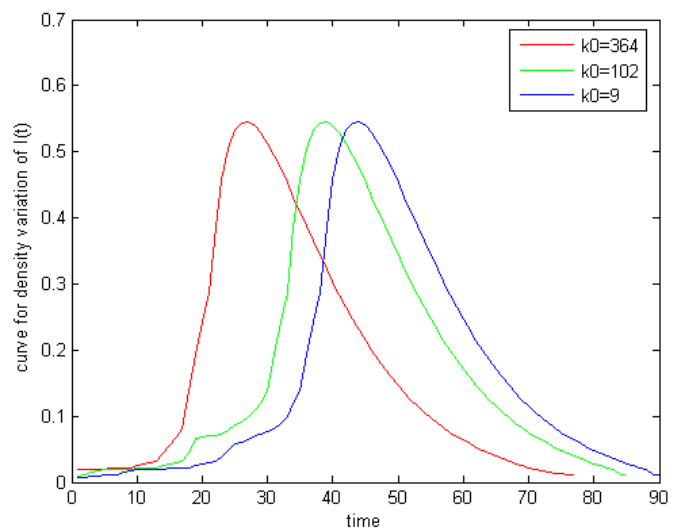

Figure 4. Influence of different initial spreading degrees on the density of spreading

\subsubsection{The influence of the degree of initial spreading nodes on spreading process}

In Figure 4, it is shown that the density of the spreading nodes $I(t)$ varies over time on the condition that the degrees of initial spreading nodes, $\mathrm{k}_{0}$, have different values. From Figure 4 , we can see that information spreads quickly in social networks when $\mathrm{k}_{0}$ has a large value. Information spreads relatively slowly when $\mathrm{k}_{0}$ has a small value. Therefore, at the beginning of advertising spreading, the choice of the core communication node (the size of the degree) has an impact on spreading speed, but has little effect on spreading rules.

\subsubsection{The influence of internal infection probability PSI on spreading process}

In Figure 5, the curve indicates that when the probability of $\mathrm{P}_{\mathrm{SI}}$ is different, $\mathrm{I}(\mathrm{t})$ varies over time. From Figure 5, we can see that with an increase in $\mathrm{P}_{\mathrm{SI}}$, the information spreading speed in the network is gradually accelerated. However, due to the small number of initial spreading nodes chosen in the initial spreading period, there is no obvious difference in spreading speed with an increase in $\mathrm{P}_{\text {SI. }}$

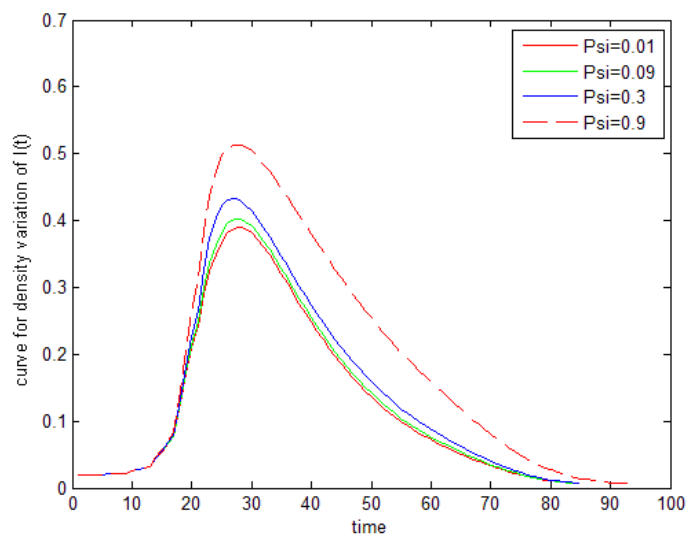

Figure 5. Density curves of spreading nodes with different $\mathrm{P}_{\mathrm{SI}}$ values

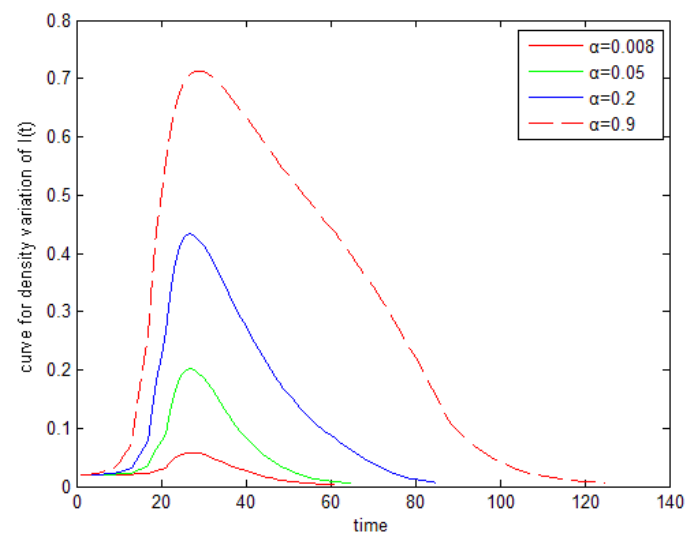

Figure 6. Curves of spreading nodes density with different a values

\subsubsection{The effect of the external infection probability $\alpha$ on spreading process}

The curve is indicated in Figure 6 when the external infection probability $\alpha$ has different values and $\mathrm{I}(\mathrm{t})$ varies over time. $\alpha$ refers to information resources for users, and it is the probability that the product information can be acquired and purchased 
through external surroundings, such as news media or shopping malls. Figure 6 clearly shows that the trend for spreading node density is obviously enhanced with an increase in $\alpha$ value, and the greater $\alpha$ value, the greater the density of spreading nodes. In addition, in the reality of social networks, the value of $\alpha$, which is generally about 0.2 , is quite reasonable, which means when $\alpha$ equals 0.2 , the indicator AEI has reached the maximum value.

\subsubsection{The effect of the direct recovery probability PSR on spreading process}

The curve in Figure 7 indicates that when the direct recovery probability $\mathrm{P}_{\mathrm{SR}}$ has different values, the density of spreading nodes $\mathrm{I}(\mathrm{t})$ varies over time. $\mathrm{P}_{\mathrm{SR}}$ is the probability that a node is directly transformed from State $\mathrm{S}$ to State R. It is clearly shown in Figure 7 that with an increase in PSR value, the density of spreading nodes has a downward trend because the greater the probability that a node transforms from State $S$ to $R$, the smaller the probability that it transforms from State $S$ to I.

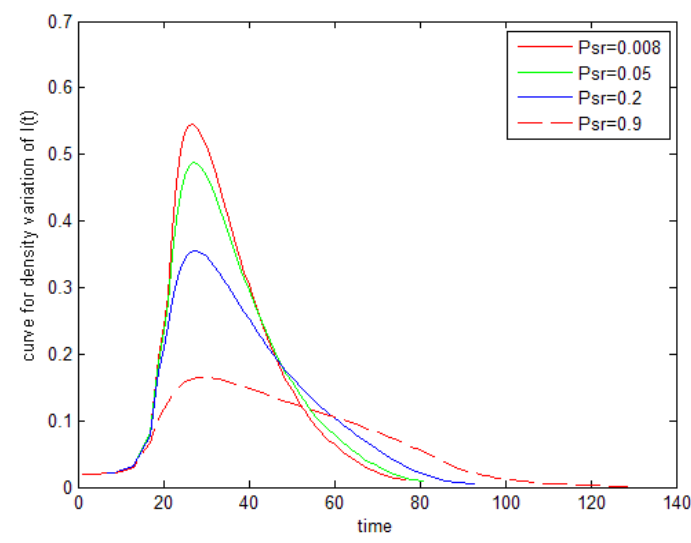

Figure 7. Density curves of spreading nodes with different PSR values

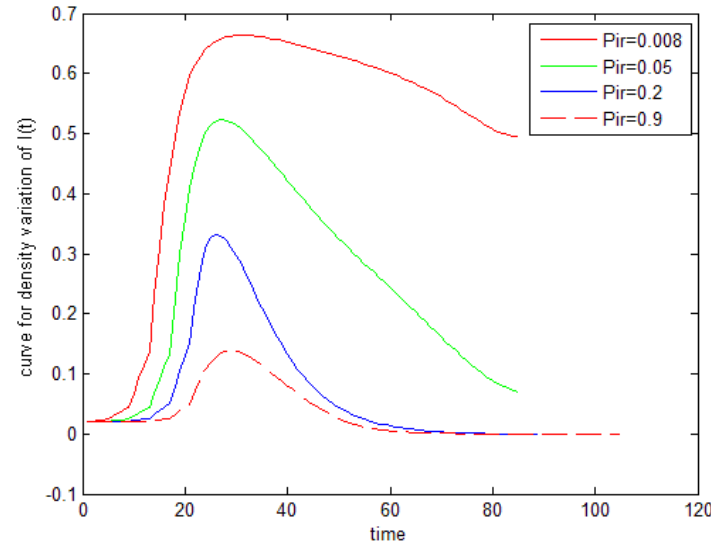

Figure 8. Density curves of spreading nodes density with different PIR values

\subsubsection{The influence of recovery probability PIR on spreading process}

In Figure 8, the curve is shown that with different values of $\mathrm{P}_{\mathrm{IR}}$, the density of spreading node $\mathrm{I}(\mathrm{t})$ varies with time. Figure 8 clearly shows that in the early spreading period, the growth rate of node density in State I does not change rapidly with an increase of $\mathrm{P}_{\mathrm{IR}}$ value due to the small number of nodes in State I. In the middle of spreading, the increasing trend is enhanced, and in the late spreading period, the transition speed of nodes in State I caused by $\mathrm{P}_{\mathrm{IR}}$ is obviously fast, namely, the larger the $\mathrm{P}_{\mathrm{IR}}$ value is, the faster the nodes in State I transform into the $\mathrm{R}$ nodes.

\section{3 the validity of the model}

At the beginning of the advertising promotion process, in order to increase the advertising coverage as soon as possible, the degree of the initial spreading node is one of the factors that determines whether it is an initial communicator. In the reference data, the largest node degree in the Facebook network is 538, which can be chosen as the initial communicator, namely $\mathrm{k}_{0}=538$; the nodes with a degree of 538 are made into State I in the network before calculation. Now, the probability of internal infection $\mathrm{P}_{\mathrm{SI}}=0.3$ and the external infection probability $\alpha=0.2$; recovery probability $\mathrm{P}_{\mathrm{IR}}=0.1$ and the direct recovery probability $\mathrm{P}_{\mathrm{SR}}=0.1$.

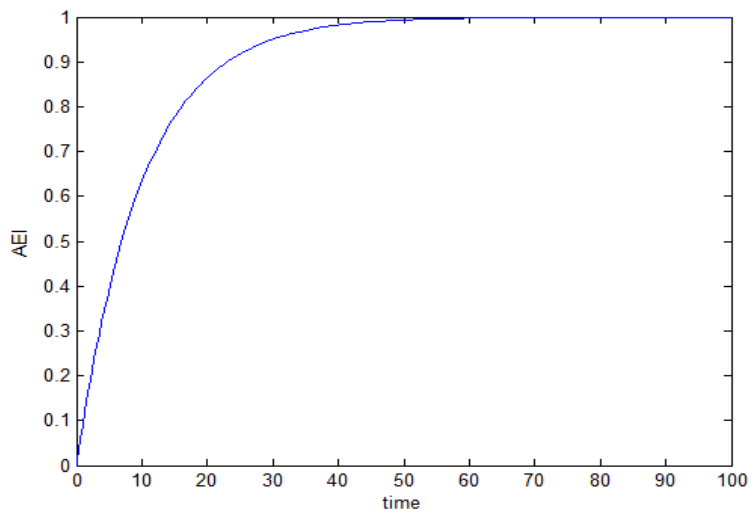

Figure 9. the chart of the AEI value changing over time 
Then, by using the iterations of Formula 5 and Formula 6, the values of A, B, C, D over time (the number of iterations is set for 100 times in this case) are obtained, and according to the model-solving method, the numbers of people in various states in the advertising promotion period are acquired. Then, the AEI formula is used to calculate the effective value of the model, and the results are shown in Figure 9.

As can be seen from Figure 9, because the largest node is chosen as the initial communicator, the effectiveness of the model rises faster in the initial period. As time goes by, the state of the system keeps stable, and the AEI value is maintained relatively stable, which demonstrates that the model proposed in this paper is effective.

\section{Conclusions}

In this paper, based on the characteristics of information propagation in social networks, the SIR model of scale-free networks is improved by two factors, which are direct recovery probability and external infection probability. At the same time, to solve the problem of a lack of unified evaluation standard for the validity of propagation models, the advertising effect index AEI is proposed as an evaluation standard to evaluate the effectiveness of advertising spreading models in social networks. With the data set obtained from Facebook, the simulation experiment and analysis on the improved models are conducted and the quantitative experiments on the model's validity are carried out. The simulation results show as follows:

(1) In the initial stage of spreading, the densities of susceptible nodes, spreading nodes and recovery nodes in the network shows a rapid rise or decay; when the evolutionary time tends to infinity, the densities of susceptible nodes, spreading nodes and recovery nodes in the network tend to be stable until it reaches a state of equilibrium. The process above is consistent with the evolution of information in real society.

(2) The choice of the initial spreading node affects the spreading speed of information in the social network. If the degree of the initial spreading node is large, which means it has more neighbor nodes, then the spreading speed of topic information in the network is fast. If the degree of the initial spreading node is small, namely it has less neighbor nodes, then the spread in the network information lags behind. The process above is basically consistent with the process of information dissemination in social networks.

(3) The AEI index shows that the advertising coverage is not "the larger, the better". Only on the condition that the parameters are reasonable does the node density in State I reach the maximum value.

\section{Acknowledgements}

This work is partially supported through grants from the National Natural Science Foundation of China (61373148 and 61502151), Shandong Province Natural Science Foundation (ZR2014FL010), Science Foundation of Ministry of Education of China (14YJC860042), Shandong Province Outstanding Young Scientist Award Fund (BS2013DX033), Shandong Province Social Sciences Project (15CXWJ13 and 16CFXJ05), and Project of Shandong Province Higher Educational Science and Technology Program (no J15LN22 and J15LN02)

\section{References}

1. X. J. Ding, "Research on Propagation Model of Public Opinion topics in SNS Based on SIR," Computer Simulation. vol. 32, no. 1, pp. 241-247, 2015 (in Chinese)

2. F. Hu, "Analysis on the Spreading Mode and Effect of Media Advertising," Media, vol.9, pp. 64-66, September 2016 (in Chinese)

3. P. Jiang and X. B. Yan, "Stability analysis and control models for rumor spreading in online social networks," International Journal of Modern Physics C, vol. 28, no. 5, May 2017

4. T. Johansson, "Gossip spread in social network Models," Physica A-Statistical Mechanics and Its Applications, vol. 471, pp. 126134, 2017

5. R. J. Li, "Research on the New Media Advertising Spreading Strategy," Media, vol. 7, pp. 63-64, July 2015 (in Chinese)

6. Q. Liu, E.H. Chen, B. Xiang, C. Ding, L. He, “Gaussian Process for Recommeiider Systems”, In 5th International Conference of Knowledge Science, Engineering and Management(KSEM ), pp. 56-67, Irvine, USA, December 2011

7. J. Tan, "Public opinion topics propagation in social network based on infectious disease model," Computer Engineering and Applications, vol. 51, no. 12, pp. 118-122, December 2015 (in Chinese)

8. Y. D. Qin, J. Ma and S. Gao, "Efficient influence maximization under TSCM: a suitable diffusion model in online social networks," Soft Computing, vol. 21, no. 4, pp. 827-838, February 2016

9. C. Tong, W.B. He, J.W. Niu and Z.Y. Xie, "A novel information cascade model in online social networks," Physica A-statistical Mechanics and Its Applications, vol. 444, pp. 297-310, February 2016 
10. J. L. Wang, F.A Liu and Z.F. Zhu, "An information spreading model based on relative weight in social network," Acta Physica Sinica, vol. 64, no. 5, pp. 050501-1- 050501-11, May 2015 (in Chinese)

11. J. Wang, Y.Q. Wang and M. "Rumor Spreading Model with Immunization Strategy and Delay Time on Homogeneous Networks," Communications in Theoretical Physics, Vol. 68, no. 6, pp. 803-810, 2017

12. Y. M. Wang and J. Wu, "The Study on Advertising Effect of Limit-up and Over-attention Underperformance," Modern Finance and Economics-Journal of Tianjin University of Finance and Economics, vol. 2, pp. 69-80, February 2015 (in Chinese)

13. A. L. Wang, W.L. Wu and J. J. Chen, "A novel rumour propagation model on social networks," International Journal of Sensor Networks, vol. 25, no.2, pp. 126-133, 2017

14. C. Wang, X.Y. Yang, K. Xu and J.F. Ma, "SEIR-Based Model for the Information Spreading over SNS," Acta Electronica Sinica, vol. 11, pp. 2325-2330, November 2014(in Chinese)

15. Y. Yang, E.H. Chen, Q. Liu, B. Xiang, T. Xu, S. Shaq, "On Approximation of Real-World Influence Spread," In the European Conference on Machine Learning and Principles and Practice of Knowledge Discovery in Databases(ECML/PKDD), pp. 548564, Bristol, UK, September 2012

16. N. Zhang, "The Spreading Strategy of TV Advertising in the New Media Era: a case study of Jiangsu TV's "Strongest Brain"," New Media Research, vol. 7, pp. 41- 48, July 2016 (in Chinese)

17. Y. C. Zhang, Y. Liu, H. F. Zhang, H. Cheng and F. Xiong, "The research of information dissemination model on online social network," Acta Physica Sinica, vol. 60, no. 5, pp. 050501-1-050501-7, May 2011 (in Chinese)

18. Y. C. Zhang, Y. Liu and Zh.J. Zhang, "Behavior and regularity analysis of online topic spreading," Journal of Electronic Science and Technology, vol. 9, no. 2, pp. 155-160, February2011 (in Chinese)

19. Y. C. Zhang, Y. Liu, H. Cheng, F. Xiong and Ch.L. Zhang, "A method of measuring user influence in microblog," Journal of Convergence Information Technology, vol. 6, no. 10, pp. 243-250, June 2011 (in Chinese)

20. Z. B. He, Z.P. Cai, J.G. Yu, X.M. Wang, Y.C. Sun and Y.S. Li, "Cost-Efficient Strategies for Restraining Rumor Spreading in Mobile Social Networks," IEEE Transactions on Vehicular Technology, vol. 66, no. 3, pp. 2789-2800, 2017

21. L. Zhu, L and Y. G. Wang, "Rumor spreading model with noise interference in complex social networks," Physica A-Statistical Mechanics and Its Applications, vol. 469, pp. 750-760, March 2017 